\title{
АПРОБАЦИЯ ТЕСТА ОБЩЕЙ ОСВЕДОМЛЕННОСТИ В РОССИИ
}

\author{
А.А. ГРИГОРЬЕВ ${ }^{\mathrm{a}}$ И.В. ЖУРАВЛЕВ ${ }^{\mathrm{b}}$, Ю.В. ЖУРАВЛЕВА \\ Е.М. ЛАПТЕВА ${ }^{\mathrm{d}}$, И.Н. НОСС
}

${ }^{a}$ ФГБУН Институт психологии РАН, 129366, Россия, Москва, ул. Ярославская, д. 13, к. 1

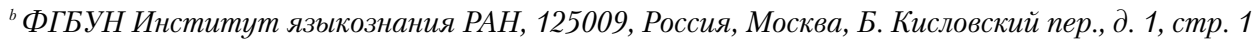

' Автономная некоммерческая организащия высшего образования «Московский гуманитарный институт имени Е.Р. Дашковой», 127349, Россия, Москва, ул. Лескова, д. 6, к. Б.

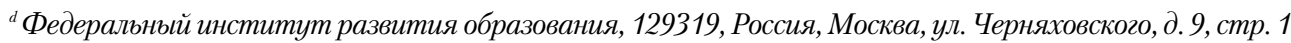

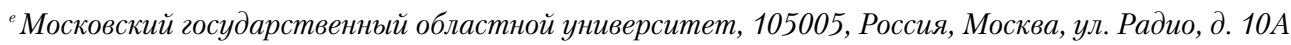

\begin{abstract}
Резюме
В статье представлены результаты апробации на российской выборке созданного в Великобритании теста общей осведомленности, оценивающего общие знания индивида в 18 содержательных областях: «История науки», «Политика», «Спорт», «История», «Классическая музыка», «Изобразительное искусство», «Литература», «Наука», «География», «Кулинария», «Медицина», «Игры», «Открытия и изобретения», «Биология», «Фильмы», «Мода», «Экономика и финансы», «Популярная музыка». Полученные в ходе апробации данные сопоставлялись с полученными в Великобритании. Результаты показали, что российские испытуемые продемонстрировали более высокую компетентность в большинстве содержательных областей. Оказалось также, что различия трудности вопросов, относящихся к разным областям знания, являются устойчивыми: имеет место значимая корреляция полученных в настоящем исследовании показателей трудности пунктов теста для 18 содержательных областей с соответствующими показателями британского исследования. Одно из возможных объяснений данного результата основывается на предположении о различии репрезентаций в системе знаний человека. Полученные данные позволили исключить из теста слишком легкие и слишком трудные (с эксцессом больше 30) для российских испытуемых пункты. Полученная в результате исключения таких пунктов версия теста характеризуется высокой надежностью (альфа Кронбаха равна 0.956). Пункты теста, вошедшие в эту версию, были использованы для сопоставления факторных структур общей осведомленности, выявленных в британском и настоящем исследованиях. Эти факторные структуры оказались в значительной степени подобными, что свидетельствует в пользу того, что общая осведомленность как когнитивная способность может быть разделена на более узкие способности.
\end{abstract}

Ключевые слова: общие знания, интеллект, факторная структура общей осведомленности.

Не вполне компетентные люди критиковали тесты интеллекта за то, что они якобы измеряют уровень знаний, а не умственные способности. Это, безусловно, не так в отношении тестов интеллекта вообще. 
Такая критика несправедлива еще и потому, что, как сейчас признается большинством специалистов, количество знаний, которыми располагает человек, имеет отношение к интеллекту. Признание этого выражается в том, что субтест общей осведомленности включен в состав некоторых имеющих хорошую репутацию тестов интеллекта (например, шкалы Векслера). Имеются солидные эмпирические свидетельства того, что общие знания являются немаловажной составляющей интеллекта. Факторная нагрузка субтеста «Осведомленность» одной из шкал Векслера, WISC-R, по фактору g (генеральному фактору интеллекта) составляет 0.68 (см.: Rushton, 1999). Это одна из высоких нагрузок субтестов WISC-R по фактору g, выше она только у субтеста «Словарный». Высокой нагрузкой (0.70) по фактоpy g характеризуется субтест «Осведомленность» и другой шкалы Векслера, WISC-III (Ibid.). В этой шкале выше, чем у субтеста «Осведомленность», нагрузки у субтестов «Арифметический» и «Словарный», такая же она у субтеста «Сходство».

В эстонской адаптации американского теста NIT (National Intelligence Test) субтест «Осведомленность» занял, по данным, полученным в разное время, еще более почетное положение (Must et al., 2009). В данных, полученных в 1933-1936 гг., он имел самую высокую факторную нагрузку по фактору g (0.88); в данных, полученных в 1997-1998 гг., его нагрузка по фактору g также была самой высокой (0.78); наконец, в данных, полученных в 2006 г., он поделил второе и третье место с субтестом «Допол- нение предложений» (с факторной нагрузкой 0.74).

Существуют и другие свидетельства того, что общая осведомленность (синонимы: общие знания, общая информированность) является важной составляющей когнитивной способности человека (см., например: Furnham, Chamorro-Premuzic, 2006). Определяя место общей осведомленности в структуре интеллекта, специалисты относят ее к кристаллизованному интеллекту, считая ее одним из его компонентов (Lynn, Irwing, 2002; Lynn et al., 2002; Furnham, Chamorro-Premuzic, 2006). Другими компонентами кристаллизованного интеллекта являются словарные способности, понимание прочитанного, правописание, способность к усвоению иностранного языка (Lynn, Irwing, 2002).

Способность приобретать, хранить и актуализировать общие знания детерминирована преимущественно генетически. В одном близнецовом исследовании (Bratko et al., 2010) наследуемость этой способности была оценена в 0,87 , в то время как наследуемость других когнитивных способностей, оцененная в этом же исследовании, варьировала от 0.48 до 0.73 .

Актуальность проблематики общих знаний отнюдь не ограничивается сферой дифференциальной психологии. Данная проблематика не менее актуальна для когнитивной психологии, в которой исследуются общие закономерности репрезентации знаний у человека.

Таким образом, постановка задачи всесторонней диагностики общей осведомленности представляется 
вполне оправданной. И эта задача была поставлена. Так как такие средства диагностики, как субтест шкал Векслера, адресуются лишь к маленькой выборке из гигантского количества знаний, которыми может обладать человек, были предприняты попытки создать тесты, обеспечивающие более полный охват знаний человека (Rolfhus, Ackerman, 1999; Irwing et al., 2001; Lynn, Irwing, 2002; Lynn et al., 2002). В одном из этих тестов (Irwing et al., 2001; Lynn, Irwing, 2002; Lynn et al., 2002) оцениваются знания в 18 областях: «Естественные науки», «Политика», «История» и т.д. (см. описание теста ниже). С помощью этого теста (в дальнейшем ТОЗ: Тест общих знаний) изучалась факторная структура общей осведомленности, сравнивалась осведомленность мужчин и женщин в различных областях (Irwing et al., 2001; Lynn et al., 2002). Вопросу о соотношении знаний мужчин и женщин авторами теста, по-видимому, придавалось особенно большое значение; включались вопросы из таких областей, как «Мода» и «Кулинария», с целью, надо полагать, избежать «смещения» теста в пользу мужчин.

В исследованиях общей осведомленности, насколько нам известно, до сих пор напрямую не ставилась задача выяснить причины различной трудности вопросов в используемых тестах общей осведомленности, в частности вопросов, относящихся к разным областям знания. А результаты, полученные с помощью ТОЗ, свидетельствуют о различии охваченных тестом областей знаний в этом отношении. Например, в среднем давалось большее количество правильных ответов на вопросы из области «Биология», чем на вопросы из области «История». Конечно, это можно объяснить неодинаковой сложностью вопросов в двух выборках материала, но возможно и другое объяснение: может быть в чем-то различной репрезентация знаний из разных областей. Полученные в последние десятилетия экспериментальные данные дают основание предположить, что объекты, входящие в разные лексико-семантические категории, по-разному репрезентированы в системе знаний человека, например, животные репрезентированы не так, как инструменты (см., например: Vitali et al., 2005). Почему бы не предположить нечто подобное в отношении знаний из различных областей?

Оценке правдоподобия того или другого объяснения должна предшествовать проверка устойчивости различий трудности вопросов, относящихся к разным областям знания. Эту устойчивость можно оценить, в частности, проведя исследования на новой выборке респондентов. В настоящей работе мы предприняли такое исследование на российской выборке. Помимо оценки устойчивости различий трудности вопросов, относящихся к разным областям знания, его данные позволят оценить и устойчивость факторной структуры общей осведомленности, что имеет значение для исследований структуры способностей человека. Возможным практическим «выходом» исследований с тестом ТОЗ было бы внедрение в практику российских психологов русскоязычной версии данного теста, адресующегося к достаточно репрезентативной выборке общих знаний. 


\section{Метод}

Испытуемые. В настоящей работе рассматриваются данные, полученные от студентов одного из московских коммерческих вузов, учащихся старших классов средней школы и учащихся заочной магистратуры Московского государственного областного университета, работников разных организаций и пенсионеров. Всего в исследовании участвовали 220 человек в возрасте 15-76 лет (в 14 случаях возраст не был указан), 95 из них были идентифицированы как мужчины, 114 как женщины, в 11 случаях пол не был указан и его не удалось определить по другим сведениям.

Tecm. TO3 в оригинальной версии состоит из 216 пунктов (вопросов), относящихся к 18 областям, по 12 вопросов на каждую. Эти области следующие: «История науки», «Политика», «Спорт», «История», «Классическая музыка», «Изобразительное искусство», «Литература», «Наука» ${ }^{1}$, «География», «Кулинария», «Медицина», «Игры», «Открытия и изобретения», «Биология», «Фильмы», «Мода», «Экономика и финансы», «Популярная музыка». Примеры вопросов: «Кто открыл “двойную спираль” ДНК?»; «Как называется столица Кении?» На каждый вопрос испытуемые должны давать свободный короткий ответ. Каждый правильный ответ оценивается в 1 балл, в некоторых случаях учитывается частичный ответ, который оценивается 0.5 балла. Некоторые вопросы допускают два правильных (альтернативных) ответа, любой из которых оценивается в 1 балл.

При переводе теста ${ }^{2}$ было обнаружено, что отдельные вопросы характеризуются явным культурным смещением и что некоторые вопросы или ответы устарели (между созданием теста и его переводом прошло более 10 лет). Такие «проблемные» пункты теста встречались в областях «Мода», «Экономика и финансы» и некоторых других. В замене или исключении некоторых вопросов, а также замене ответов или допущении альтернативных ответов на некоторые вопросы состояла первичная адаптация теста. Прошедшая первичную адаптацию русскоязычная версия ТОЗ была использована для сбора данных.

Процедура. ТОЗ предъявлялся в бланковой форме. На выполнение теста был отведен 1 час.

\section{Результаты и обсуждение}

При обработке полученных данных обнаружилось, что не все испытуемые проходили тест до конца, некоторые не пытались ответить на все вопросы. Таких испытуемых оказалось 25, их ответы были изъяты. Ответы остальных 195 испытуемых были подвергнуты обработке и анализу. Кроме того, из областей «Мода» и «Популярная музыка» были исключены по два вопроса, относительно ответов на которые возникли сомнения.

\footnotetext{
${ }^{1}$ В английском варианте теста - «General science», т.е. общенаучные сведения.

${ }^{2}$ Выражаем благодарность профессору Р. Линну, одному из авторов теста, снабдившему нас необходимыми материалами.
} 
На первом шаге обработки было рассчитано среднее количество правильных ответов на один пункт теста для каждой из 18 областей. Полученные величины вместе с соответствующими значениями для данных, полученных Р. Линном с соавт. (Lynn et al., 2002), приведены в таблице 1.

Просматривая значения в таблице 1 , можно увидеть, что в 11 областях испытуемые из наших выборок оказались «компетентнее» испытуемых Линна с соавт., последние же продемонстрировали бо́льшую осве- домленность в семи остальных областях. Однако четыре из них - те, в которых можно подозревать наличие временно́го (эпохального, «поколенного») или культурного смещения: «Спорт», «Фильмы», «Мода», «Популярная музыка». Действительно, на такие вопросы, как «Какая страна выиграла чемпионат мира по футболу в 1998 г.?» или «Какой цвет стал хитом сезона в 1997 г.?», было гораздо вероятней получить правильный ответ около 2000 г., чем в 2014 г., тем более от людей, которые родились в 1997-1998 гг. (а таких среди наших

Таблица 1

Среднее число правильных ответов на один пункт теста для 18 областей

\begin{tabular}{|l|l|c|}
\hline \multicolumn{1}{|c|}{ Область } & Наши данные & $\begin{array}{c}\text { Данные Линна и др. } \\
\text { (Linn et al., 2002) }\end{array}$ \\
\hline История науки & 0.221 & 0.134 \\
\hline Политика & 0.214 & 0.188 \\
\hline Спорт & 0.276 & 0.428 \\
\hline История & 0.315 & 0.128 \\
\hline Классическая музыка & 0.062 & 0.026 \\
\hline Изобразительное искусство & 0.133 & 0.083 \\
\hline Литература & 0.225 & 0.087 \\
\hline Наука & 0.401 & 0.337 \\
\hline География & 0.242 & 0.063 \\
\hline Кулинария & 0.321 & 0.466 \\
\hline Медицина & 0.526 & 0.546 \\
\hline Игры & 0.374 & 0.482 \\
\hline Открытия и изобретения & 0.167 & 0.086 \\
\hline Биология & 0.464 & 0.448 \\
\hline Фильмы & 0.101 & 0.224 \\
\hline Мода & 0.154 & 0.503 \\
\hline Экономика и финансы & 0.425 & 0.291 \\
\hline Популярная музыка & 0.121 & 0.422 \\
\hline
\end{tabular}


испытуемых было немало). Все фильмы, по которым задаются вопросы в тесте, американские или английские. Возможным временны́м «смещением», однако, может характеризоваться и одна область, в которой наши испытуемые показали лучшую осведомленность, - «Политика». Если исключить все эти области, соотношение осведомленности по областям станет более «однонаправленным»: 10 против 3.

Рассмотрим теперь вопрос об устойчивости различий трудности вопросов, относящихся к разным областям знания. Во-первых, определим, существуют ли значимые различия между областями общих знаний в наших данных. Дисперсионный анализ по всем 18 областям знаний дал следующие результаты: $\mathrm{F}=134.34$; $\mathrm{df}=17$ и 3492; $p<0.001$. Дисперсионный анализ по 13 областям знаний, в которых наличие смещения менее ожидаемо, дал следующие результаты: $\mathrm{F}=125.95 ; \mathrm{df}=12$ и 2522; $p<0.001$. Таким образом, трудность вопросов, относящихся к разным областям знания, различается значимо. Во-вторых, рассмотрим, в какой мере различия в двух рядах данных наших и Линна с соавт. - соответствуют друг другу. Коэффициент корреляции по всем 18 областям знаний составляет 0.545 ( $p<0.05)$, исключение пяти, вероятно, «смещенных» областей повышает его до 0.857 $(p<0.001)$. Эти результаты согласуются с предположением о существовании устойчивых различий трудности вопросов, относящихся к разным областям знания. Их объяснение - задача будущих исследований.

Выше говорилось о нашем стремлении внедрить в практику россий- ских психологов русскоязычную версию ТОЗ, чьи пункты адресовались бы к достаточно репрезентативной выборке общих знаний. Это, разумеется, предполагает адаптацию теста. Его первичная адаптация была проведена до сбора данных (см. выше). Дальнейшая адаптация должна была быть осуществлена на основе собранных данных. Следовало, очевидно, выявить слишком трудные и слишком легкие пункты теста. С этой целью был рассчитан эксцесс каждого пункта. Если эксцесс превышает 30, то пункт является или слишком трудным, или слишком легким. Пунктов с эксцессом, не превышающим 30, оказалось 172. Эти пункты составили русскоязычную версию теста. Данная версия характеризуется высокой внутренней согласованностью (альфа Кронбаха равна 0.956). Мы, однако, не считаем ее окончательной: она скорее должна рассматриваться как предварительная версия, на основе которой может быть создана окончательная.

Последним шагом нашего анализа было сопоставление факторной структуры общей осведомленности, выявленной в работе Ирвинга с соавт. (Irwing et al., 2001), с нашими данными. С этой целью мы сделали факторный анализ наших данных, ориентируясь на проведенные Ирвингом с соавт. процедуры. Эти авторы, во-первых, подвергли факторному анализу вопросы, относящиеся к каждой из 18 областей. Все области, кроме одной, оказались гомогенными; единственная негомогенная область - «Популярная музыка» была разделена ими на «Популярную музыку» и «Джаз». Таким образом, областей общих знаний стало 19. 
Во-вторых, ими был проведен исследовательский (эксплораторный) факторный анализ для 19 областей. Втретьих, они провели конфирматорный (подтверждающий) факторный анализ для 19 областей.

С целью обеспечить соответствие анализа мы вслед за Ирвингом с соавт. разделили вопросы, относящиеся к области «Популярная музыка», на две части, а затем провели исследовательский факторный анализ для 19 областей, следуя процедуре этих авторов (факторы выделялись методом максимального правдоподобия с последующим облиминвращением). Анализу была подвергнута версия теста из 172 вопросов.

В то время как у Ирвинга с соавт. выделилось пять факторов с собственным значением больше единицы, у нас таких факторов выделилось четыре. Для удобства соотнесения состава выделившихся факторов мы ограничились рассмотрением в каждом факторе трех областей, имеющих по нему максимальные по абсолютной величине нагрузки (в одном из факторов, выделившихся в нашем исследовании, - четырех областей по причине того, что нагрузки, занимающие третье и четвертое места, оказались одинаковыми). Факторные структуры, полученные Ирвингом с соавт. и нами, приведены в таблице 2; значения нагрузок заменены их рангами в порядке убывания абсолютной величины.

При просмотре данных таблицы 2 обращает на себя внимание, во-первых, сходство первого фактора в наших данных с пятым фактором Ирвинга с соавт. - фактором компетентности в сфере искусства. Во-вторых, определенное сходство имеет наш второй фактор с первым фактором Ирвинга с соавт., относящимся к компетенции в общественно-политической области. В-третьих, наш третий фактор соотносим со вторым фактором Ирвинга с соавт., фактором компетентности в таких областях, как «Мода» и «Популярная музыка». Наконец, с нашим четвертым фактором имеет сходство третий фактор Ирвинга с соавт., который может быть с некоторой натяжкой интерпретирован как компетентность в домоводстве. Факторный анализ наших данных не выделил фактора, который бы соответствовал четвертому фактору Ирвинга с соавт., относящемуся к компетентности в таких областях, как «Игры» и «Ппорт».

Таким образом, в целом наблюдается сходство факторных структур данных двух исследований. Это сходство свидетельствует о том, что общая осведомленность как когнитивная способность может быть разделена на более узкие способности. Данный результат является важным для дифференциальной психологии. Для когнитивной психологии больший интерес представляет подтверждение устойчивости различий трудности вопросов, относящихся к разным областям знания. Как уже говорилось выше, одно из возможных объяснений этого - различие репрезентаций в системе знаний человека. Проверка возможных объяснений - задача будущих работ.

Версия теста из 172 пунктов, к которой мы пришли в данной работе, может быть взята за основу при подготовке окончательной версии теста. При подготовке окончательной версии следует руководствоваться, на 
Результаты исследовательского факторного анализа

\begin{tabular}{|c|c|c|c|c|c|c|c|c|c|}
\hline \multirow[t]{3}{*}{ Область } & \multicolumn{9}{|c|}{ Факторы } \\
\hline & \multicolumn{5}{|c|}{ Ирвинг с соавт. } & \multicolumn{4}{|c|}{ Наши данные } \\
\hline & 1 & 2 & 3 & 4 & 5 & 1 & 2 & 3 & 4 \\
\hline \multicolumn{10}{|l|}{ История науки } \\
\hline Политика & 1 & & & & & 3.5 & 3 & & \\
\hline Спорт & & & & 2 & & & & & \\
\hline История & 2 & & & & & & 1 & & \\
\hline Классическая музыка & & & & & 1 & 1 & & & \\
\hline Изобразительное искусство & & & & & 3 & 3.5 & & & \\
\hline Литература & & & & & 2 & 2 & & & \\
\hline Наука & & & 3 & & & & & & \\
\hline \multicolumn{10}{|l|}{ География } \\
\hline Кулинария & & & 1 & & & & & 3 & 2 \\
\hline Медицина & & & 2 & & & & & & 1 \\
\hline Игры & & & & 1 & & & & & \\
\hline Открытия и изобретения & & & & & & & 2 & & \\
\hline Биология & & & & 3 & & & & & 3 \\
\hline Фильмы & & 3 & & & & & & & \\
\hline Мода & & 1 & & & & & & 2 & \\
\hline Экономика и финансы & 3 & & & & & & & & \\
\hline \multicolumn{10}{|l|}{ Джаз } \\
\hline Популярная музыка & & 2 & & & & & & 1 & \\
\hline
\end{tabular}

наш взгляд, следующими соображениями. Во-первых, не имеет смысла включать в тест пункты, которые могут потребовать ежегодного пересмотра; более того, нецелесообразно сохранять представительство в тесте областей знаний, чреватых подоб- ным динамизмом. Во-вторых, нецелесообразно оставлять в тесте слишком легкие пункты. Однако, в-третьих, наличие там особо трудных пунктов может оказаться полезным как средство диагностики выдающейся одаренности.

\section{Литература}

Bratko, D., Butkovic, A., \& Chamorro-Premuzic, T. (2010). The genetics of general knowledge: A twin study from Croatia. Personality and Individual Differences, 48, 403-407. 
Furnham, A., \& Chamorro-Premuzic, T. (2006). Personality, intelligence and general knowledge. Learning and Individual Differences, 16(1), 79-90.

Irwing, P., Cammock, T., \& Lynn, R. (2001). Some evidence for the existence of a general factor of semantic memory and its components. Personality and Individual Differences, 30(5), 857-871.

Lynn, R., \& Irwing, P. (2002). Sex differences in general knowledge, semantic memory and reasoning ability. British Journal of Psychology, 93(4), 545-556.

Lynn, R., Irwing, P., \& Cammock, T. (2002). Sex differences in general knowledge. Intelligence, 30(1), 27-40.

Must, O., te Nijenhuis, J., Must, A., \& van Vianen, A. E. M. (2009). Comparability of IQ scores over time. Intelligence, 37(1), 25-33.

Rolfhus, E. L., \& Ackerman, P. L. (1999). Assessing individual differences in knowledge: Knowledge, intelligence, and related traits. Journal of Educational Psychology, 91(3), 511-526.

Rushton, J. P. (1999). Secular gains in IQ not related to the $\mathrm{g}$ factor and inbreeding depression - unlike Black-White differences: A reply to Flynn. Personality and Individual Differences, 26, 381-389.

Vitali, P., Abutalebi, J., Tettamanti, M., Rowe, J., Scifo, P., Fazio, F., ... Perani, D. (2005). Generating animal and tool names: An fMRI study of effective connectivity. Brain and Language, 93(1), 32-45.
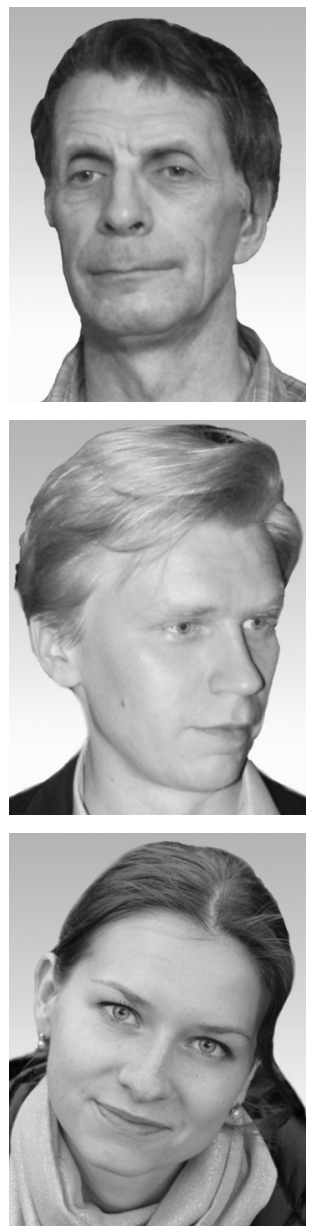

Григорьев Андрей Александрович - главный научный сотрудник, ФГБУН Институт психологии РАН, доктор филологических наук, доцент.

Сфера научных интересов: интеллект, индивидуальные различия, психолингвистика.

Контакты: andrey4002775@yandex.ru

Журавлев Игнатий Владимирович - старший научный сотрудник, ФГБУН Институт языкознания РАН, кандидат психологических наук.

Сфера научных интересов: психолингвистика, мышление, сознание, когнитивные процессы, социальные когнитивные функции.

Контакты: semiotik@yandex.ru

Журавлева Юлия Валерьевна - старший преподаватель, автономная некоммерческая организация высшего образования «Московский гуманитарный институт имени Е.Р. Дашковой».

Сфера научных интересов: психолингвистика, социальные когнитивные функции, мифологическое мышление.

Контакты: jv.clinic@yandex.ru 


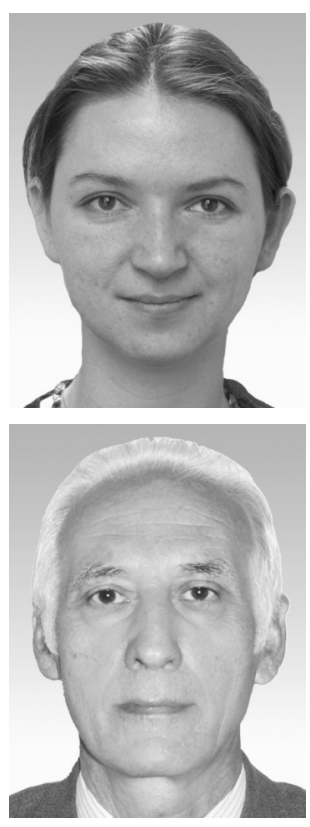

Лаптева Екатерина Михайловна - ведущий научный сотрудник, Федеральный институт развития образования, кандидат психологических наук.

Сфера научных интересов: интеллект, креативность, когнитивные механизмы способностей.

Контакты: ek.lapteva@gmail.com

Носс Игорь Николаевич - профессор кафедры психологии труда и организационной психологии, Московский государственный областной университет, доктор психологических наук, профессор. Сфера научных интересов: профессиональная психодиагностика. Контакты: innoss2007@yandex.ru

\title{
Approbation of the Test of General Knowledge in Russia
}

\author{
A.A. Grigoriev ${ }^{a}$, I.V. Zhuravlev ${ }^{b}$, J.V. Zhuravleva ${ }^{c}$, E.M. Lapteva ${ }^{d}$, I.N. Noss ${ }^{e}$ \\ ${ }^{a}$ Institute of Psychology of Russian Academy of Sciences, 13 build. 1, Yaroslavskaya str., Moscor, 129366, \\ Russian Federation \\ ${ }^{b}$ Institute of Linguistics of Russian Academy of Sciences, 1 Bolshoi Kislorsky lane, Moscor, 125009, \\ Russian Federation \\ 'Moscow Humanities Institute named E. R. Dashkova, 6 b Leskova Str., Moscow, 127349, Russian Federation \\ ${ }^{d}$ Federal Institute of Education Development, $9 / 1$ Chernyakhovskogo Str., Moscow, 125319, Russian Federation \\ ${ }^{e}$ Moscow State Regional University, 10 Radio Str., Moscow, 105005, Russian Federation
}

\begin{abstract}
The article presents the results of approbation on the Russian sample of the general knowledge test developed in UK. The test estimates general knowledge of an individual in 18 domains: History of Science; Politics; Sport; History; Classical Music; Art; Literature; General Science; Geography; Cookery; Medicine; Games; Discovery and Exploration; Biology; Film; Fashion; Finance; Popular Music. The obtained data was compared with the UK data. The results show that the Russian participants have higher competence in most domains. Also, it was shown that differences in difficulty of questions in various domains are stable: there is a significant correlation between measures of difficulty of the test items for the 18 domains in the Russian and the British data. One possible explanation of this result relies on the hypothesis of differences in representations in the system of human knowledge. The obtained data have enabled us to exclude from the test too easy and too difficult (with kurtosis more than 30) items for the Russian participants. The version of the test after this
\end{abstract}


exclusion is highly reliable (the Cronbach's alpha is 0.956). The test items, included in this version, were used for the comparison of the factor structures of general knowledge revealed in the British and the present studies. These factor structures were rather similar. This confirms that general knowledge as a cognitive ability can be partitioned into more narrow abilities.

Keywords: general knowledge, intelligence, factor structure of general knowledge.

\section{References}

Bratko, D., Butkovic, A., \& Chamorro-Premuzic, T. (2010). The genetics of general knowledge: A twin study from Croatia. Personality and Individual Differences, 48, 403-407.

Furnham, A., \& Chamorro-Premuzic, T. (2006). Personality, intelligence and general knowledge. Learning and Individual Differences, 16(1), 79-90.

Irwing, P., Cammock, T., \& Lynn, R. (2001). Some evidence for the existence of a general factor of semantic memory and its components. Personality and Individual Differences, 30(5), 857-871.

Lynn, R., \& Irwing, P. (2002). Sex differences in general knowledge, semantic memory and reasoning ability. British Journal of Psychology, 93(4), 545-556.

Lynn, R., Irwing, P., \& Cammock, T. (2002). Sex differences in general knowledge. Intelligence, 30(1), 27-40.

Must, O., te Nijenhuis, J., Must, A., \& van Vianen, A. E. M. (2009). Comparability of IQ scores over time. Intelligence, 37(1), 25-33.

Rolfhus, E. L., \& Ackerman, P. L. (1999). Assessing individual differences in knowledge: Knowledge, intelligence, and related traits. Journal of Educational Psychology, 91(3), 511-526.

Rushton, J. P. (1999). Secular gains in IQ not related to the $\mathrm{g}$ factor and inbreeding depression - unlike Black-White differences: A reply to Flynn. Personality and Individual Differences, 26, 381-389.

Vitali, P., Abutalebi, J., Tettamanti, M., Rowe, J., Scifo, P., Fazio, F., ... Perani, D. (2005). Generating animal and tool names: An fMRI study of effective connectivity. Brain and Language, 93(1), 32-45.

Andrei A. Grigoriev - chief research fellow, Institute of Psychology of Russian Academy of Sciences, D.Sc., associate professor.

Research area: intelligence, individual differences, psycholinguistics.

E-mail: andrey4002775@yandex.ru

Ignatiy V. Zhuravlev - senior research fellow, The Institute of Linguistics RAS, Ph.D.

Research area: psycholinguistics, thinking, consciousness, cognition, social cognitive functions.

E-mail: semiotik@yandex.ru

Julia V. Zhuravleva - senior lecturer, Moscow Humanities Institute named E.R. Dashkova.

Research area: psycholinguistics, social cognitive functions, mythological thinking.

E-mail: jv.clinic@yandex.ru

Ekaterina M. Lapteva - leading research fellow, Federal Institute of Education Development, Ph.D. Research area: intelligence, creativity, cognitive mechanisms of abilities

E-mail: ek.lapteva@gmail.com

Igor N. Noss - professor, Department of psychology organizational and work psychology, Moscow State Regional University, D.Sc.

Research area: professional psychodiagnostics.

E-mail: innoss2007@yandex.ru 